\title{
Down-regulation of miR-146b-5p by long noncoding RNA MALAT1 in hepatocellular carcinoma promotes cancer growth and metastasis
}

\author{
Chao Li ${ }^{1}$, Runchen Miao ${ }^{1}$, Sushun Liu ${ }^{1}$, Yong Wan ${ }^{2}$, Simin Zhang ${ }^{1}$, Yan Deng ${ }^{1}$, \\ Jianbin $\mathrm{Bi}^{1}$, Kai $\mathbf{Q u}^{1}$, Jingyao Zhang ${ }^{1}$, Chang Liu ${ }^{1}$ \\ ${ }^{1}$ Department of Hepatobiliary Surgery, The First Affiliated Hospital of Xi'an Jiaotong University, Xi'an 710061, Shaanxi \\ Province, China \\ ${ }^{2}$ Department of Geriatric Surgery, The First Affiliated Hospital of Xi'an Jiaotong University, Xi'an 710061, Shaanxi Province, China \\ Correspondence to: Chang Liv, email: liuchangdoctor@163.com \\ Keywords: microRNA-146b-5p, hepatocellular carcinoma, TNF receptor associated factor 6, growth, metastasis \\ Received: December 28, $2016 \quad$ Accepted: January 25, $2017 \quad$ Published: February 23, 2017 \\ Copyright: Li et al. This is an open-access article distributed under the terms of the Creative Commons Attribution License \\ (CC-BY), which permits unrestricted use, distribution, and reproduction in any medium, provided the original author and source \\ are credited.
}

\section{ABSTRACT}

MicroRNAs play an important role in liver cancer genesis and progression. In this study, we identified down-regulation of miR-146b-5p associated with tumor growth, metastasis and poor survival in hepatocellular carcinoma (HCC) patients. miR-146b-5p could suppress proliferation, migration, and invasion and induced apoptosis in vitro and in vivo. Remarkably, TNF receptor associated factor 6 (TRAF6) was confirmed as a direct target of miR-146b-5p in HCC and miR-146b-5p exerted the tumor suppression roles through inhibiting the phosphorylation of Akt mediated by TRAF6. Furthermore, we identified long non-coding RNA MALAT1 as a molecular sponge of miR-146b-5p to down-regulate its expression in HCC. In general, our results indicate that miR146b-5p inhibits tumor growth and metastasis of HCC by targeting TRAF6 mediated Akt phosphorylation.

\section{INTRODUCTION}

Hepatocellular carcinoma (HCC) is one of the most common digestive cancers in China [1]. MicroRNAs represent as a class of endogenous short single-chain RNAs that regulate various cellular processes through binding to the 3' untranslated region (3'-UTR) of target mRNAs [2]. Plenty of reports have well established their roles in metabolism [3], growth [4], senescence [5], angiogenesis [6] and metastasis [7].

Data in mounting numbers revealed that miR-146b$5 p$ was involved in cancer genesis and progression [8]. However, miR-146b-5p is a friend or foe that depends on the cancer types. Up-regulation of miR-146b-5p was found in osteosarcoma tissues [9], and it promoted proliferation, migration and invasion through inhibiting ZNRF3 in osteosarcoma cells. Nevertheless, miR-146b$5 \mathrm{p}$ inhibited glioma cells' migration and invasion through silencing MMP-16 expression [10]; it also attenuated stemness and radioresistance through restraining $\mathrm{HuR} /$ lincRNA-p21/ $\beta$-catenin pathway [11]. In papillary thyroid carcinoma (PTC), the role of miR-146b-5p was more equivocal. An elevated expression of miR-146b-5p was observed in PTC tissues $[12,13]$. Over-expression of miR$146 b-5 p$ enhanced growth and metastasis of PTC cells $[14,15]$. However, recent study discovered that exosomes derived from TPC-1 cells were rich in miR-146b-5p, but these exosomes served as a negative regulator for cell proliferation to both TPC-1 and normal thyroid follicular cells [16]. Even the role of miR-146b-5p has been uncovered in many cancers, the expression and functions of miR-146b-5p in HCC are still unclear.

In this study, we disclosed that miR-146b-5p was down-regulated in $\mathrm{HCC}$ tissues and correlated with poor prognosis. In vitro and in vivo experiments showed that miR-146b-5p could suppress proliferation, migration, and invasion and induce apoptosis through inhibiting TRAF6/ p-Akt signaling pathway. 


\section{RESULTS}

\section{Decreased expression of miR-146b-5p is associated with malignant clinical features and poor prognosis}

As shown in Figure 1A and 1B, $44 \mathrm{HCC}$ tissues $(73.33 \%)$ were detected down-regulated $\left(\log ^{\mathrm{NT} / \mathrm{T}}>0\right)$ miR-146b-5p expression, whereas only 17 HCC tissues had up-regulated $\left(\log ^{\mathrm{NT} / \mathrm{T}}<0\right)$ miR-146b-5p expression $(P<0.001)$. We selected the mean level of miR-146b-5p (mean value $=1.917$ ) as a cut-off value to divide all 60 HCC patients into miR-146b-5p low expression group $(\mathrm{n}=35,<1.917)$ and high expression group $(\mathrm{n}=25$, $\geq 1.917$ ). As shown in Table 1, decreased expression of miR-146b-5p was associated with large tumor diameter ( $\geq 5 \mathrm{~cm}, P=0.033$ ), venous infiltration $(P=0.046)$ and advanced TNM stage (stage III+IV, $P=0.040$ ). In this cohort, compared with the patients in high miR-146b-5p expression group, patients in low miR-146b-5p expression group had low 5-year overall survival (OS) rate (logrank $=5.606, P=0.018$, Figure $1 \mathrm{C}$ ) and disease-free survival (DFS) rate (log-rank $=7.692, P=0.005$, Figure 1D).
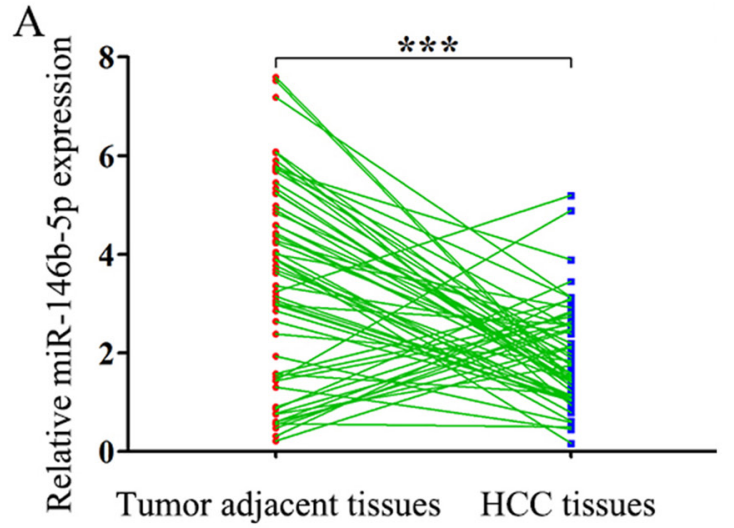

$\mathrm{C}$

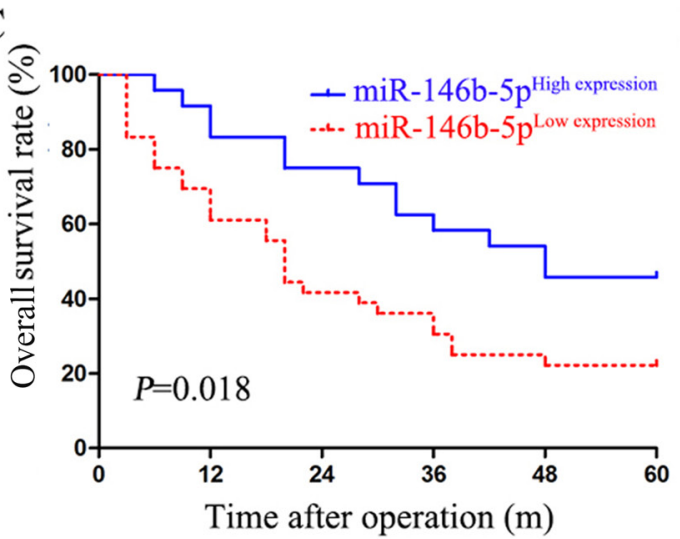

miR-146b-5p inhibits growth and metastasis in $\mathrm{HCC}$ cells

We detected the expression levels of miR-146b-5p in four HCC cell lines (MHCC97-H, SMMC-7721, Hep3B and HepG2) and a human immortal liver cell line (LO2). As shown in Figure 2A, compared with the expression of miR-146b-5p in LO2, MHCC97-H and Hep3B had the lowest and highest expression levels of miR-146b-5p in these four cell lines, repectively $(P<0.001$, respectively). So we selected these two cell lines for further experiments. First, we stably over-expressed miR-146b-5p in MHCC97-H cells and knocked down miR-146b-5p expression in Hep3B cells $(P<0.001$, respectively, Figure $2 \mathrm{~B})$. Next, we concluded from CCK-8 assays and plate clone formation assays that miR-146b-5p over-expression suppressed cell viability and proliferation in MHCC97-H cells; on the contrary, inhibition of miR-146b-5p depressed cell viability and proliferation in Hep3B cells $(P<0.01$, respectively, Figure $2 \mathrm{C}$ and $2 \mathrm{D}$ ). Additionally, determined by flow cytometry and caspase 3/7 activity assays, up-regulation of miR146b-5p induced apoptosis in MHCC97-H cells and downregulation of miR-146b-5p reduced apoptosis in Hep3B
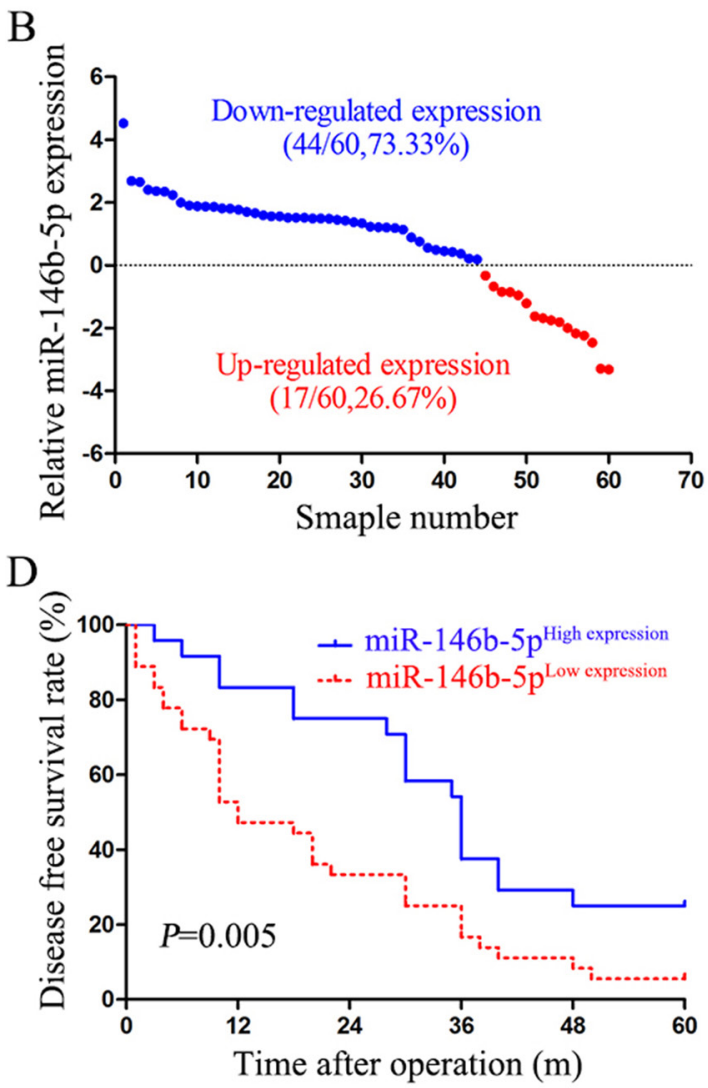

Figure 1: Low expression of miR-146b-5p in HCC tissues relates to poor prognosis. A. The expression levels of miR-146b$5 \mathrm{p}$ in HCC tissues and matched tumor-adjacent tissues. ${ }^{* * *} P<0.001$. B. Down-regulation of miR-146b-5p was detected in $73.33 \%(44 / 60)$ of HCC tissues. C and D. Low expression of miR-146b-5p was significantly associated with the poorer 5-year overall survival (C, logrank=5.606, $P=0.018)$ and disease-free survival $(\mathrm{D}, \log$-rank=7.692, $P=0.005)$ of HCC patients. Patients were divided into high and low expression subgroups according to the mean value of miR-146b-5p. 
Table 1: Clinical correlation of $\mathrm{miR}-146 \mathrm{~b}-5 \mathrm{p}$ expression in $\mathrm{HCC}(\mathrm{N}=60)$

\begin{tabular}{|c|c|c|c|c|c|c|}
\hline \multirow{2}{*}{ Clinical Characteristics } & & \multirow{2}{*}{$\begin{array}{l}\text { No. of patients } \\
(N=60)\end{array}$} & \multicolumn{2}{|c|}{ No. of patients $(N=60)$} & \multirow{2}{*}{$\chi^{2}$} & \multirow{2}{*}{$P$} \\
\hline & & & $146 b-5 p^{\mathrm{High}} n=25$ & $146 b-5 p^{\text {Low }} n=35$ & & \\
\hline \multirow[t]{2}{*}{ Gender } & Male & 39 & 15 & 24 & \multirow{2}{*}{0.471} & \multirow{2}{*}{0.493} \\
\hline & Female & 21 & 10 & 11 & & \\
\hline \multirow{2}{*}{ Age (year) } & $<50$ & 29 & 11 & 18 & \multirow{2}{*}{0.322} & \multirow{2}{*}{0.570} \\
\hline & $\geq 50$ & 31 & 14 & 17 & & \\
\hline \multirow{2}{*}{ Tumor size $(\mathrm{cm})$} & $<5$ & 24 & 14 & 10 & \multirow{2}{*}{4.571} & \multirow{2}{*}{$0.033^{*}$} \\
\hline & $\geq 5$ & 36 & 11 & 25 & & \\
\hline \multirow{2}{*}{ Liver cirrhosis } & Absent & 13 & 7 & 6 & \multirow{2}{*}{1.013} & \multirow{2}{*}{0.314} \\
\hline & Present & 47 & 18 & 29 & & \\
\hline \multirow{2}{*}{ Serum AFP level (ng/mL) } & $<400$ & 20 & 11 & 9 & \multirow{2}{*}{2.194} & \multirow{2}{*}{0.139} \\
\hline & $\geq 400$ & 40 & 14 & 26 & & \\
\hline \multirow{2}{*}{ Venous infiltration } & Absent & 42 & 21 & 21 & \multirow{2}{*}{4.000} & \multirow{2}{*}{$0.046^{*}$} \\
\hline & Present & 18 & 4 & 14 & & \\
\hline \multirow{2}{*}{$\begin{array}{l}\text { Edmondson-Steiner } \\
\text { grading }\end{array}$} & $\mathrm{I}+\mathrm{II}$ & 35 & 11 & 24 & \multirow{2}{*}{3.623} & \multirow{2}{*}{0.057} \\
\hline & $\mathrm{III}+\mathrm{IV}$ & 25 & 14 & 11 & & \\
\hline \multirow{2}{*}{ TNM stage } & $\mathrm{I}+\mathrm{II}$ & 39 & 20 & 19 & \multirow{2}{*}{4.239} & \multirow{2}{*}{$0.040^{*}$} \\
\hline & III+IV & 21 & 5 & 16 & & \\
\hline
\end{tabular}

${ }^{*} P<0.05$.

Abbreviations: AFP, alpha-fetoprotein; TNM, tumor-node-metastasis.

cells $(P<0.01$, respectively, Figure $2 \mathrm{E}$ and $2 \mathrm{~F})$. We further determined cell migration and invasion through transwell chambers. As shown in Figure 2G, up-regulation of miR$146 \mathrm{~b}-5 \mathrm{p}$ significantly decreased the migration and invasion in MHCC97-H cells $(P<0.01$, respectively). On contrary, down-regulation of miR-146b-5p increased the migration and invasion in Hep3B cells $(P<0.01$, respectively).

\section{miR-146b-5p inhibits tumor growth and metastasis in vivo}

In order to further confirm our in vitro results, we subcutaneously injected the recombinant MHCC97-H and Hep3B cells into nude mice, measured the tumor volume each week for 4 weeks and drew the tumor growth curves. As shown in Figure 3A, miR-146b-5p over-expression significantly repressed xenograft tumor growth, whereas miR-146b-5p knockdown promoted tumor growth $(P<0.01$, respectively, Figure $3 \mathrm{~A})$. Determined by $\mathrm{Ki}-$ 67 IHC staining and TUNEL assays, miR-146b-5p was demonstrated to inhibit proliferation $(P<0.01$, respectively, Figure 3B) and induce apoptosis $(P<0.01$, respectively, Figure $3 \mathrm{C}$ ). Moreover, we established the lung metastasis mice model through the tail vein injection. Lung tissue sections were stained with hematoxylin and eosin. Compared with control groups, miR-146b-5p overexpression decreased the lung metastasis but miR-146b-
$5 \mathrm{p}$ knockdown increased the lung metastasis $(P<0.01$, respectively, Figure 3D).

\section{TRAF6 is a direct target of miR-146b-5p in HCC}

As shown in Figure 4A, TNF receptor associated factor 6 (TRAF6) was selected as a potential downstream target of miR-146b-5p through five online databases (Targetscan: http://www.targetscan.org/, picTar: http:// pictar.mdc-berlin.de/, RNA22: https://cm.jefferson.edu/ rna22/Interactive/, PITA: https:/genie.weizmann.ac.il/ and miRanda: http://www.microrna.org/). We constructed a mutation type of miR-146b-5p and used dual-luciferase reporter gene assays to demonstrate that the luciferase activity of TRAF6 containing 3'-UTRs was inhibited only by the wild-type miR-146b-5p but not the mutant (mut) miR-146b-5p $(P<0.001$, Figure 4B). In vitro, we revealed that up-regulation of miR-146b-5p inhibited TRAF6 mRNA and protein expression in MHCC97-H cells, whereas downregulation of miR-146b-5p induced TRAF6 expression in Hep3B cells $(P<0.01$, respectively, Figure 4C and 4D). We also detected the mRNA and protein expression levels in nude mice subcutaneous tumor by qRT-PCR and IHC, respectively. As shown in Figure 4E and 4F, miR-146b-5p significantly inhibited TRAF6 expression in vivo $(\mathrm{P}<0.01$, respectively). Finally, we detected the expression of TRAF6 in $\mathrm{HCC}$ tissues by IHC staining. As shown in Figure 4G, 

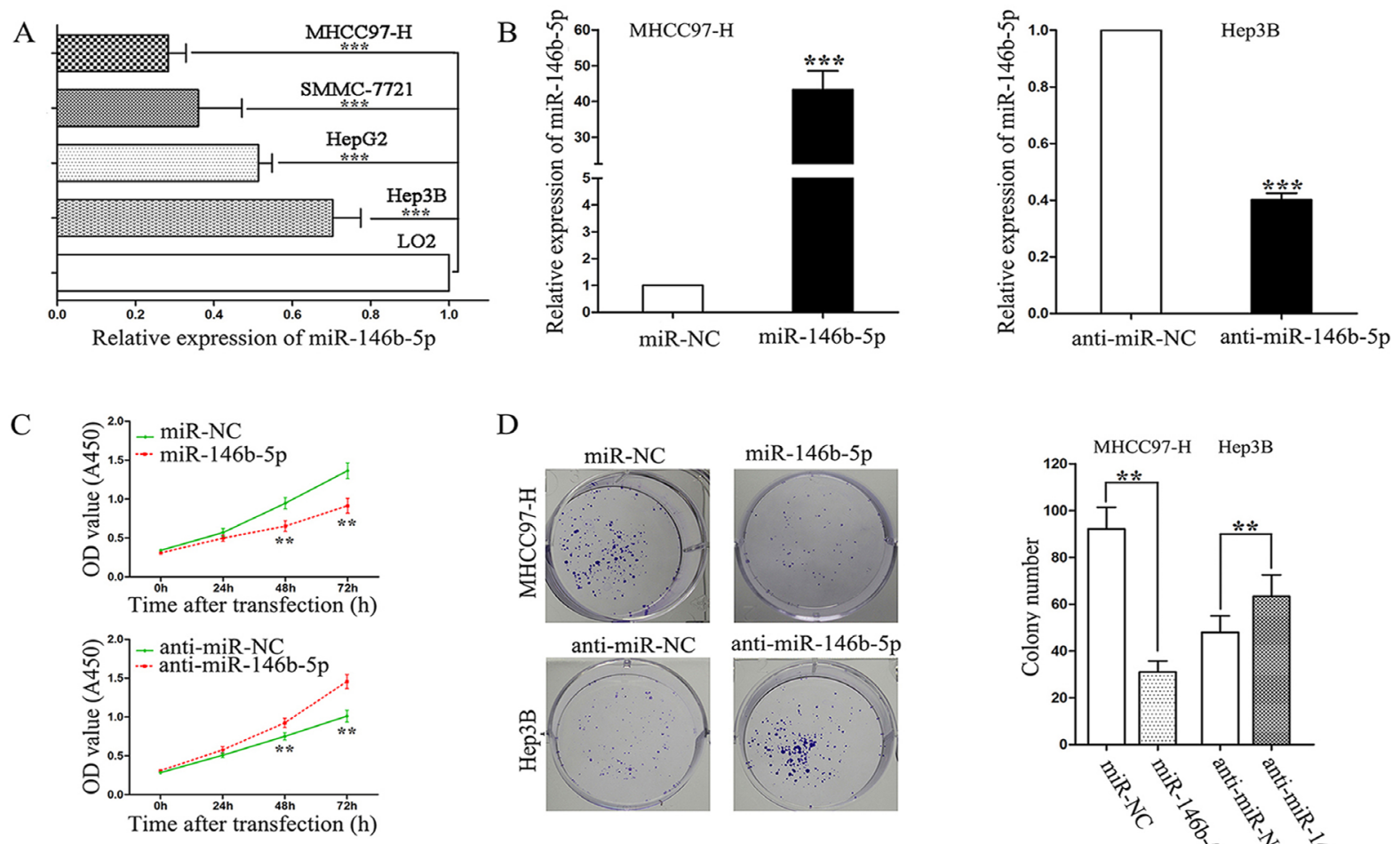

E
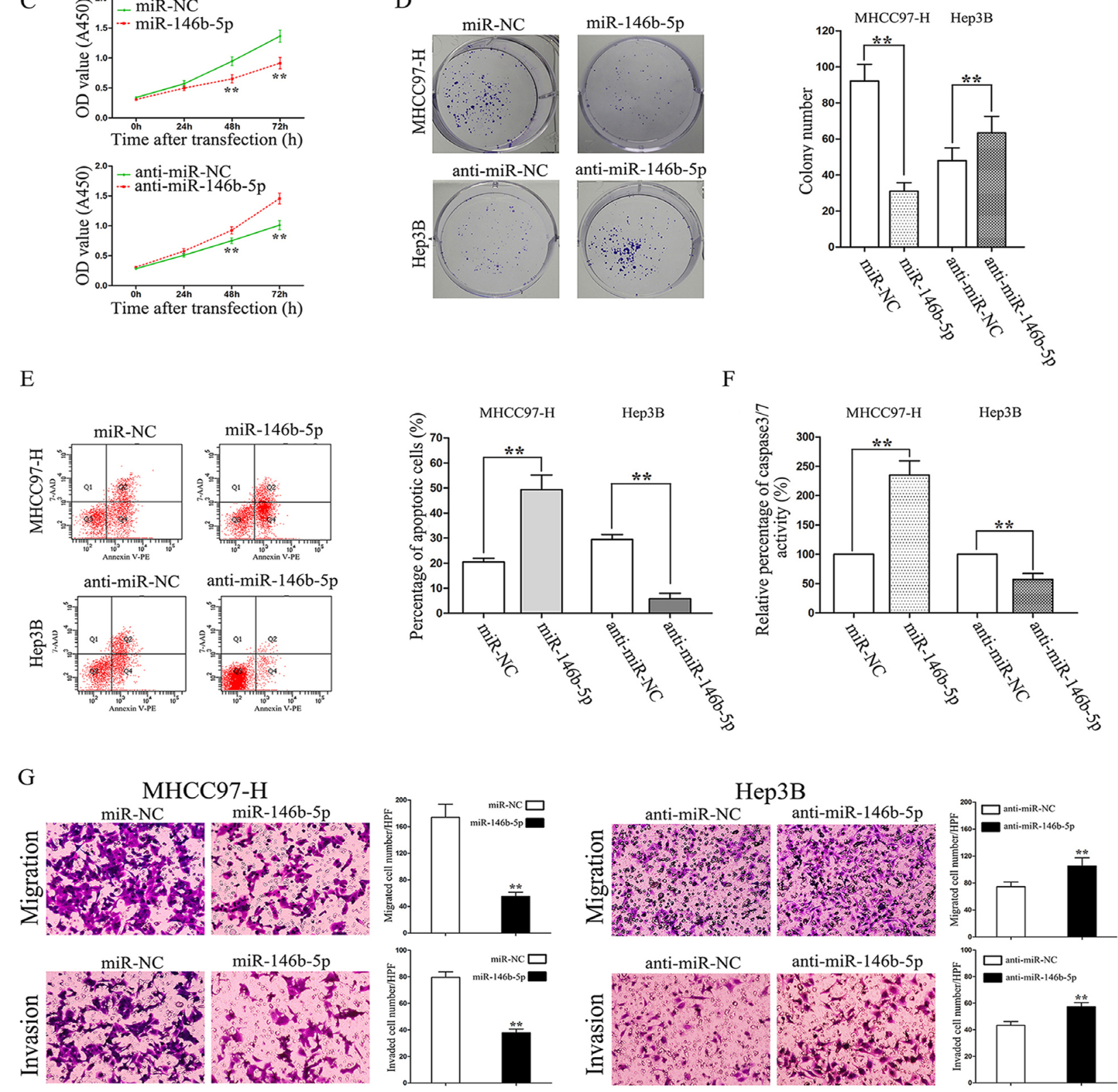

Figure 2: The effects of miR-146b-5p on proliferation, apoptosis, migration, and invasion of HCC cells. A. Expression of miR-146b-5p in the human immortal liver cell line (LO2) and four HCC cell lines (MHCC97-H, SMMC-7721, Hep3B and HepG2). ${ }^{* * *} P<0.001$. B. Alteration of miR-146b-5p levels in HCC cells according their basal levels. miR-146b-5p expression levels were determined by qRT-PCR. ${ }^{* * * *} P<0.001$. C-G. Alteration of miR-146b-5p expression levels markedly influenced cell viability (C), colony numbers (D), apoptotic cell percentages (E), caspase 3/7 enzyme activity (F), migration and invasion (G) in MHCC97-H and Hep3B cells. ${ }^{* *} P<0.01$. All experiments were performed at least in triplicate and the data in $\mathbf{A}-\mathbf{G}$ are presented as the (mean $\pm \mathrm{SD}$ ). 
the expression level of TRAF6 in the low miR-146b-5p group was higher than that in the high miR-146b-5p group $(P<0.01$, respectively, Figure $4 \mathrm{G})$.

\section{miR-146b-5p attenuates TRAF6/p-Akt signal pathway in $\mathrm{HCC}$}

TRAF6 is thought to achieve its functions partly through contributing the phosphorylation of Akt [17]. In order to explore whether miR-146b-5p performs its anti-cancer role though inhibiting the TRAF6-mediated phosphorylation of Akt, we detected the expression of total Akt (t-Akt) and phosphorylated Akt (p-Akt) in transfected cells. As shown in Figure 5, miR-146b-5p over-expression decreased the expression of p-Akt in MHCC97-H cells, whereas miR-146b-5p knockdown increased the expression of p-Akt in Hep3B cells $\left(P<0.05\right.$, respectively, $1^{\text {st }}$ row $)$. However, no significant expression changes of total Akt (t-Akt) were detected in these two cell lines $(P>0.05$, respectively, $2^{\text {nd }}$ row). Subsequently, we detected a series of downstream genes of the TRAF6/p-Akt signaling pathway, such as Bcl-2, Mcl-1 and MMP-9. Western blot results showed that the proteinlevels of these three molecules were down-regulated in MHCC97-H cells and up-regulated in Hep3B cells $\left(P<0.05\right.$, respectively, $3^{\text {rd }}$ to $5^{\text {th }}$ rows $)$.

\section{TRAF6 alters the effects of miR-146b-5p on HCC cells}

In contrast, we transfected TRAF6 vectors into miR146b-5p overexpressed MHCC97-H (MHCC97- $\mathrm{H}^{\text {miR-146b-5p }}$ ) and TRAF6 siRNAs into miR-146b-5p knockdown Hep3B cells $\left(\right.$ Hep3 $\left.3 \mathrm{~B}^{\text {anti-miR-146b-5p }}\right)$. As shown in Figure 6A, 6B and 6C, TRAF6 over-expression in MHCC97- $\mathrm{H}^{\text {miR-146b-5p }}$ cells promoted cell viability, migration, invasion and suppressed apoptosis; however, TRAF6 knockdown in Hep3B anti-miR- $^{-}$ ${ }^{1466-5 p}$ cells inhibited cell viability, migration, invasion and induced apoptosis $(P<0.05$ and $P<0.01$, respectively). Furthermore, TRAF6 abrogated the effects of miR-146b$5 \mathrm{p}$ for Akt phosphorylation as well as protein expression
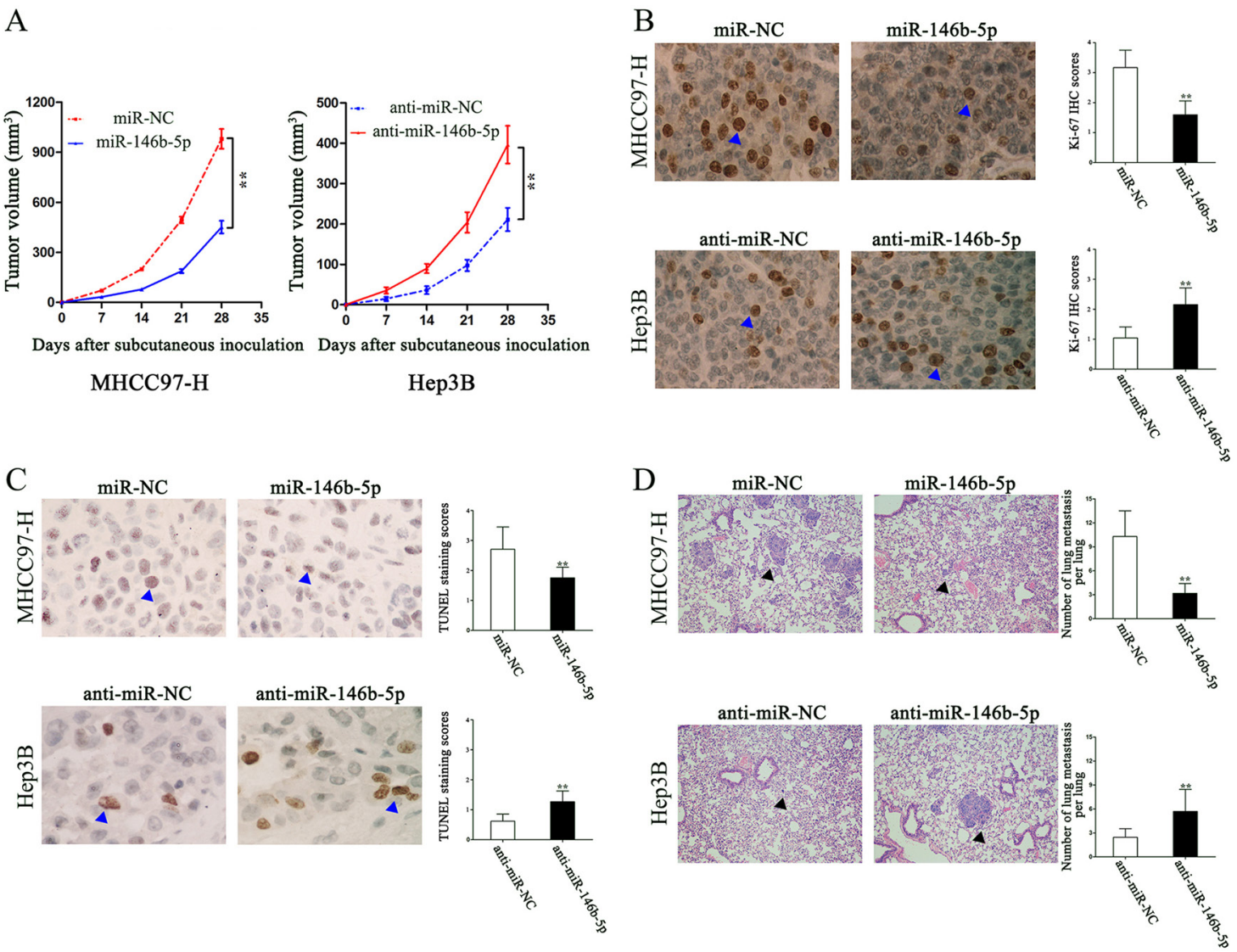

Figure 3: The in vivo effects of miR-146b-5p on tumor growth and metastasis of HCC. A. As determined by tumor growth curves, miR-146b-5p over-expression markedly suppressed subcutaneous transplantation tumor growth; however, miR-146b$5 \mathrm{p}$ promoted tumor growth. $\mathrm{n}=$ six for every group. ${ }^{* *} P<0.01$. B and C. In vivo, miR-146b-5p inhibited MHCC97-H cells proliferation and induced apoptosis, whereas anti-miR-146b-5p promoted Hep3B cells proliferation and arrested apoptosis.** $P<0.01$. D. Lung metastatic clusters performing by tail vein injection are shown at 4 weeks. Over-expression of miR-146b-5p in MHCC97-H cells decreased their lung metastasis; miR-146b-5p knockdown increased the lung metastasis of Hep3B cells. ${ }^{* *} P<0.01$. Data in A-D are presented as the (mean $\pm \mathrm{SD})$. 

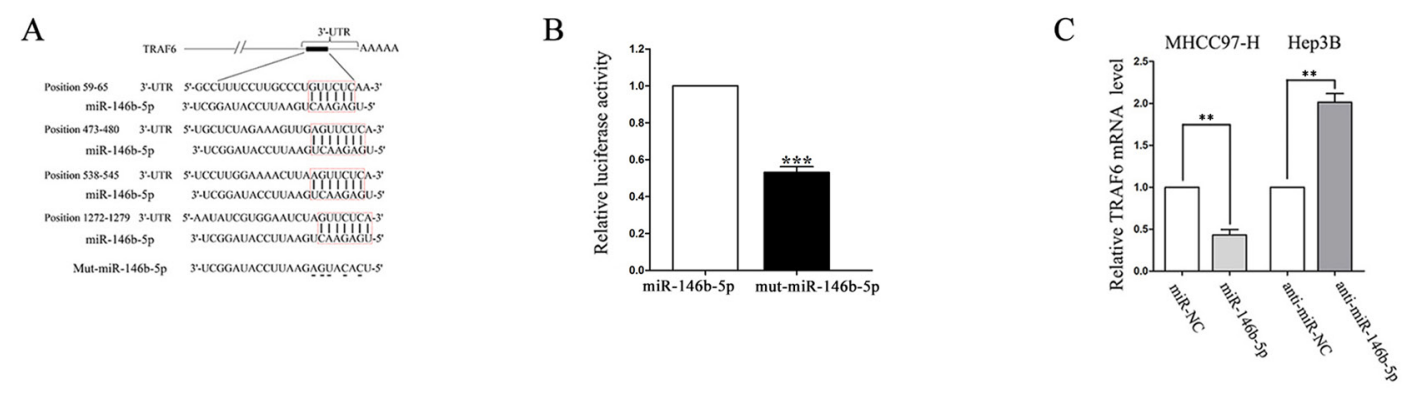

$\mathrm{D}$
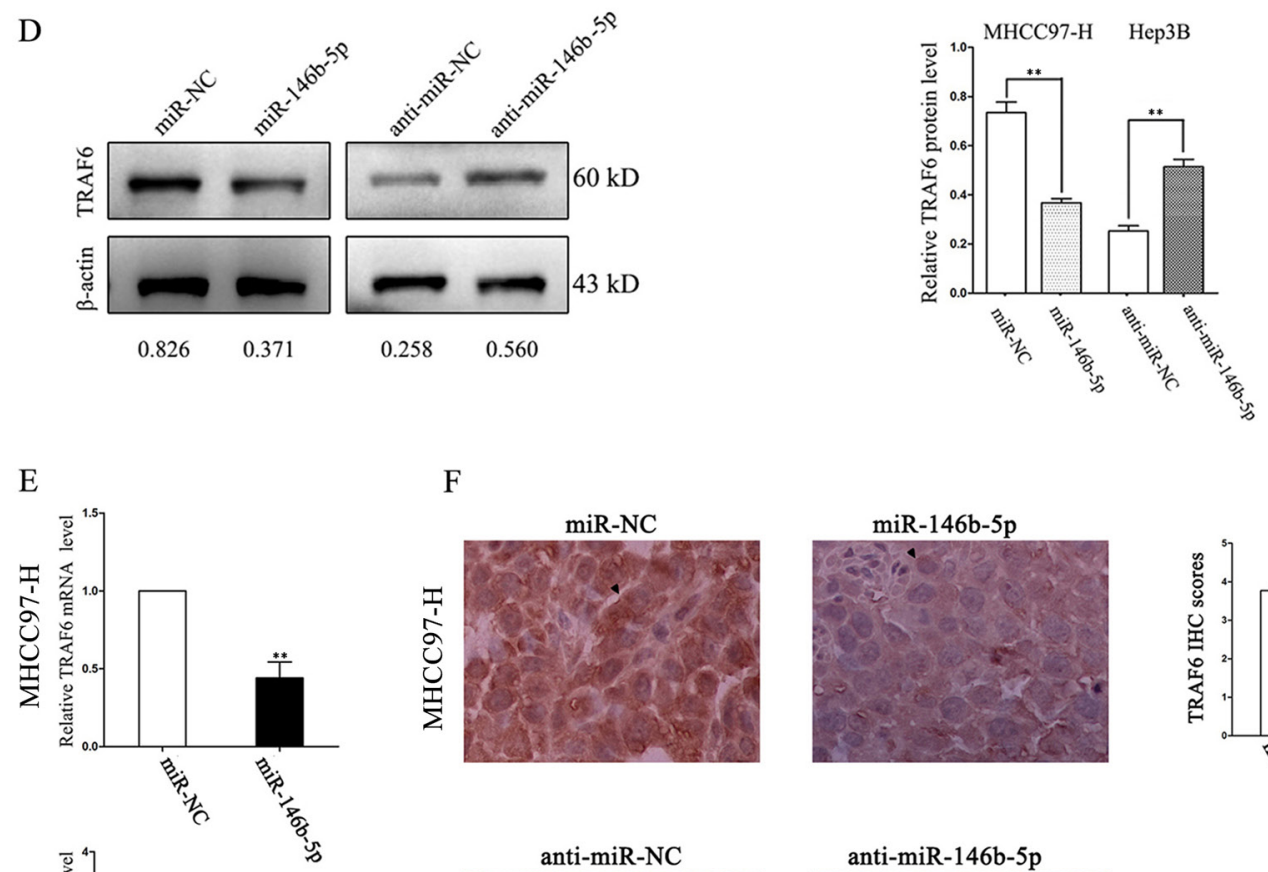

F
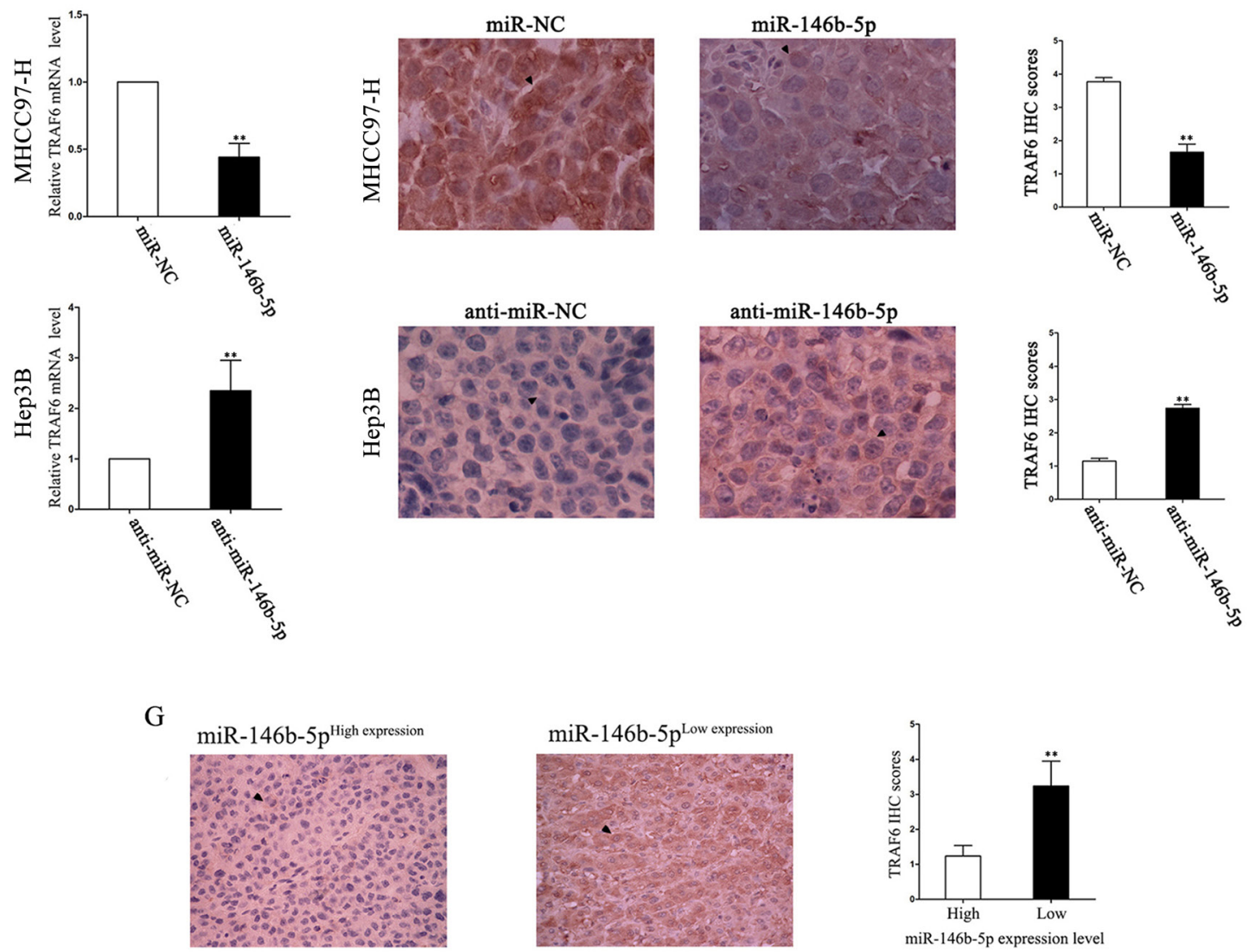

Figure 4: TRAF6 is a direct downstream target of miR-146b-5p in HCC. A. The assumed miR-146b-5p binding sequences in the 3'-UTR of TRAF6. B. Wild-type miR-146b-5p crippled the luciferase activity that carried the 3'-UTR of TRAF6 mRNA. ${ }^{* * *} P<0.001$. C-F. Alteration of miR-146b-5p levels regulated TRAF6 expression in vitro (C and D) and in vivo (E and F). The mRNA (C and E) and protein (D and F) expression levels of TRAF6 were down-regulated through miR-146b-5p over-expression and up-regulated by miR-146b$5 \mathrm{p}$ knockdown. The mRNA levels were determined by qRT-PCR. The protein expression was detected by immunoblotting in vitro and IHC in vivo. ${ }^{* *} P<0.01$. G. TRAF6 expression was lower in high miR-146b-5p group than that in low miR-146b-5p group. ${ }^{* *} P<0.01$. All experiments were performed at least in triplicate and the data in $\mathbf{B}-\mathbf{G}$ are presented as the (mean $\pm \mathrm{SD}$ ). 
of Bcl-2, Mcl-1 and MMP-9 ( $P<0.01$, respectively, Figure $6 \mathrm{D})$. However, there were no significant changes for total Akt in these two cell types $(P>0.05$, respectively, Figure 6D).

\section{Long non-coding RNA MALAT1 serves as an endogenous sponge of miR-146b-5p in HCC}

Studies have shown that long noncoding RNAs (lncRNAs) act as an endogenous sponge of microRNAs. We used Starbase v.2.0 to predict that a long non-coding RNA, metastasis-associated lung adenocarcinoma transcript 1 (MALAT1), had a complementary sequence of miR-146b-5p. We found that the expression of MALAT1 was higher in HCC tissues than that in tumor-adjacent tissues $(P<0.01$, Figure 7A). Furthermore, a negative relationship was identified between these two non-coding RNAs, the correlation index was $-0.483(P=0.007$, Figure 7B). MHCC97-H and SMMC-7721 cells which had high original MALAT1 expression levels $(P<0.01$, respectively, Figure 7C) were selected to silence MALAT1 expression by siRNA $(P<0.001$, respectively, Figure 7D). Real-time PCR results showed that MALAT1 knockdown rescued miR-146b-5p expression in MHCC97-H and SMMC-7721

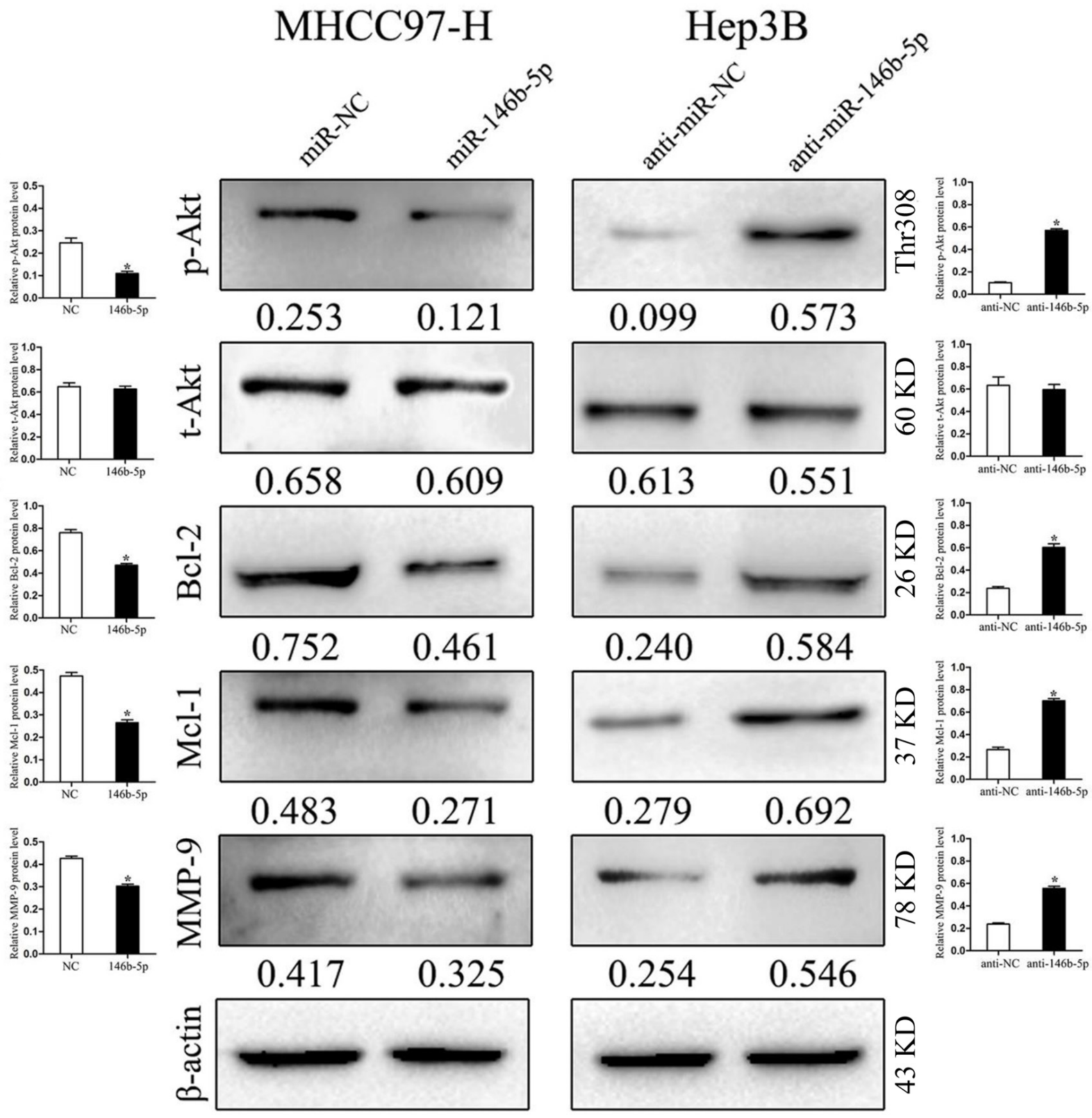

Figure 5: miR-146b-5p exerts its functions through inhibiting the TRAF6/p-Akt signaling pathway. In MHCC97-H cells with miR-146b-5p over-expression, the phosphorylation of Akt (p-Akt) was impaired and the expression levels of Bcl-2, Mcl-1 and MMP-9 were decreased. In Hep3B cells with miR-146b-5p knockdown, enhanced the phosphorylated Akt induced the expression of Bcl-2, Mcl-1 and MMP-9. Neither over-expression nor knockdown of miR-146b-5p could find any significant changes of total Akt ( $\mathrm{t}$-Akt) expression. ${ }^{*} P<0.05$. Immunoblotting was performed at least in triplicate and the data are presented as the (mean $\pm \mathrm{SD}$ ). 
cells $(P<0.001$, respectively, Figure 7E). These findings might explain why miR-146b-5p expression was lower in HCC.

\section{DISCUSSION}

miR-146b-5p showed paradoxical roles in human cancers. It was reduced in gallbladder cancer tissues and correlated with large tumor size and inferior cell differentiation [18]. A prognostic value of miR-146b-5p was also embodied in ER-negative breast cancer patients [19], large B-cell lymphoma patients [20] and gastric

A
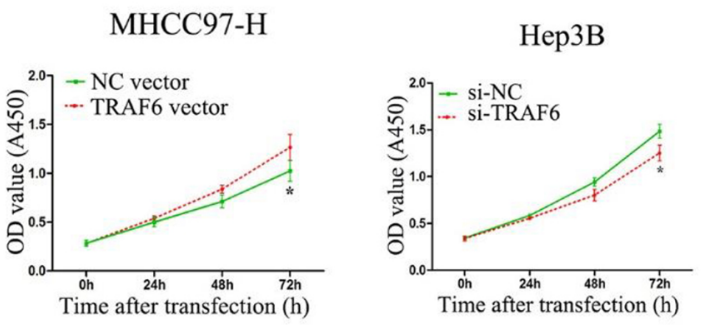

C
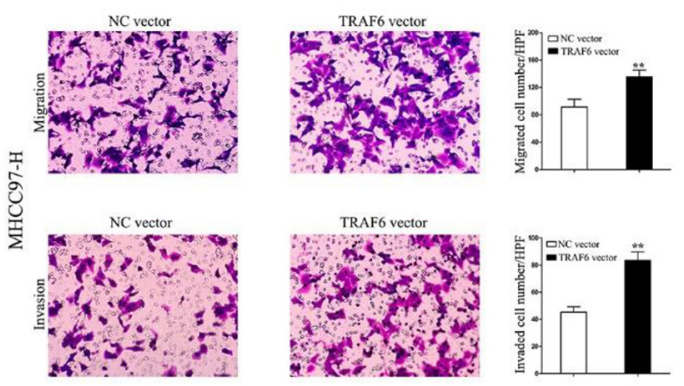

D

MHCC97-H ${ }^{\text {miR-146b-5p }}$
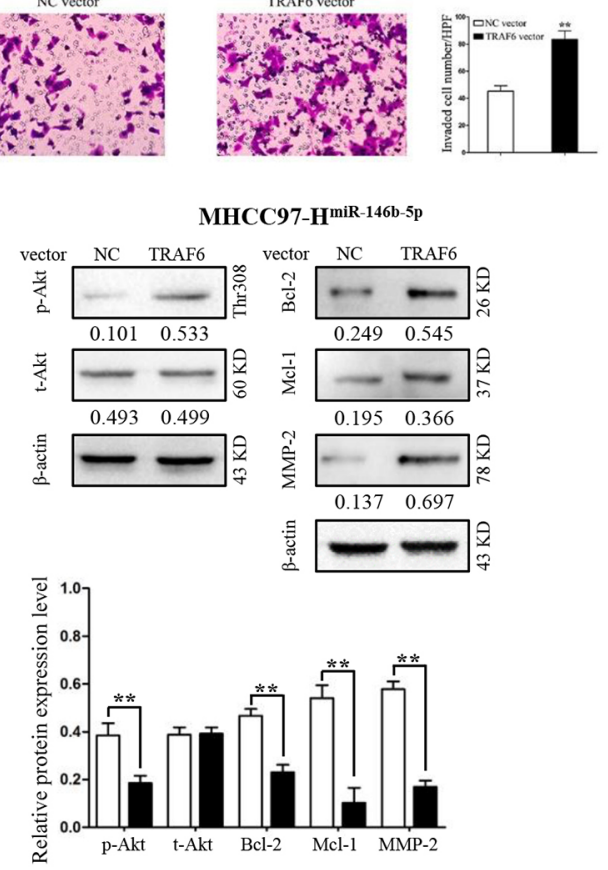

cancer [21]. Interestingly, Yoon et.al reported that high expression of miR-146b-5p in the proximal resection margin tissues of gastric cancer was a strong risk factor for recurrence and poor survival [22]. In the present study, for the first time, we discovered that the expression of miR-146b-5p was significantly decreased in HCC tissues. Down-regulation of miR-146b-5p was correlated with malignant clinical features and poor survival in HCC patients. All above results indicate that decreased expression of miR-146b-5p is an unfavorable factor and may lead to the disorder of cell growth and invasion in HCC.

B
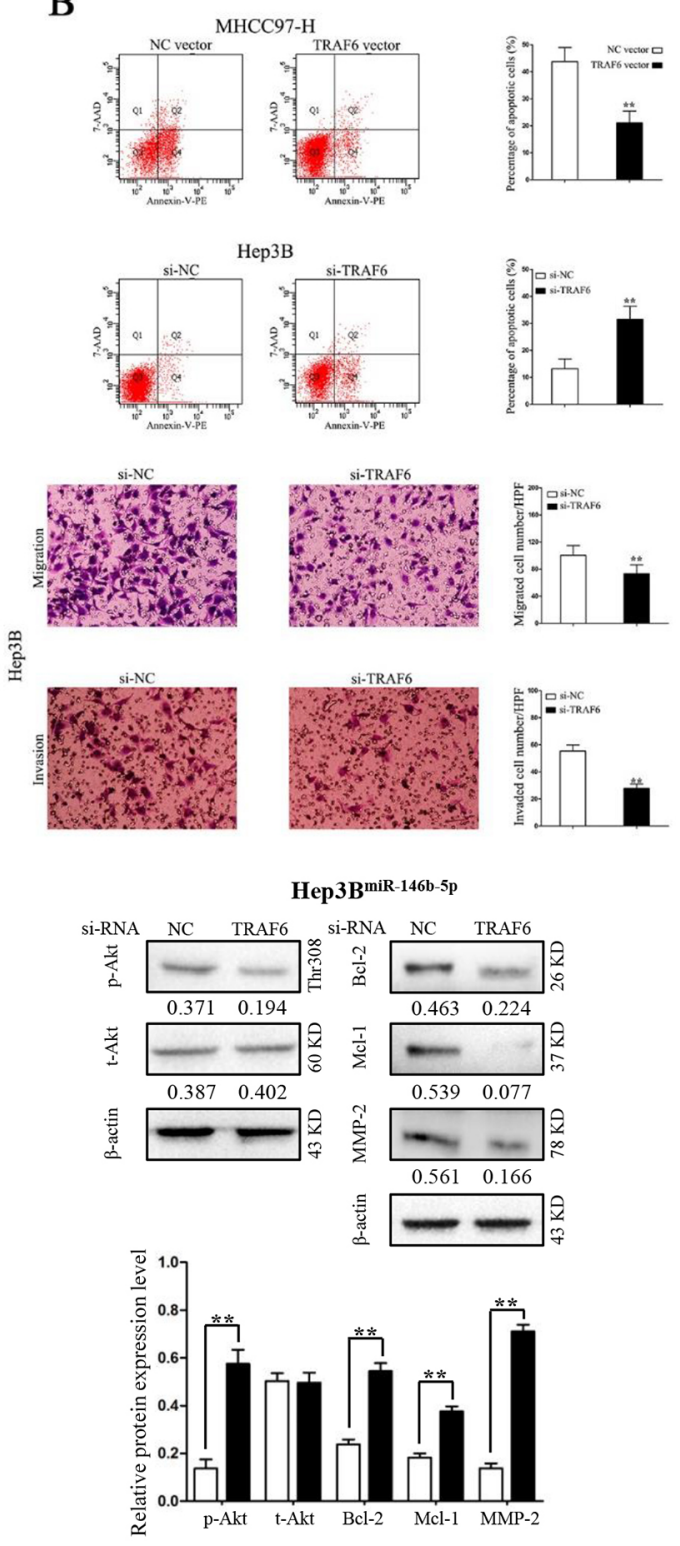

Figure 6: Modification of TRAF6 expression partly abrogated the functions of miR-146b-5p on HCC cells. A-C. Modification of TRAF6 expression partly abolished the effects of miR-146b-5p on cell viability (A), apoptosis (B), migration and invasion (C) of MHHC97-H and Hep3B cells. ${ }^{*} P<0.05,{ }^{* *} P<0.01$. D. TRAF6 abrogated the effects of miR-146b-5p for Akt phosphorylation as well as the protein levels of Bcl-2, Mcl-1 and MMP-9. ${ }^{* *} P<0.01$. All experiments were performed at least in triplicate and the data in A-D are presented as the (mean $\pm \mathrm{SD})$. 

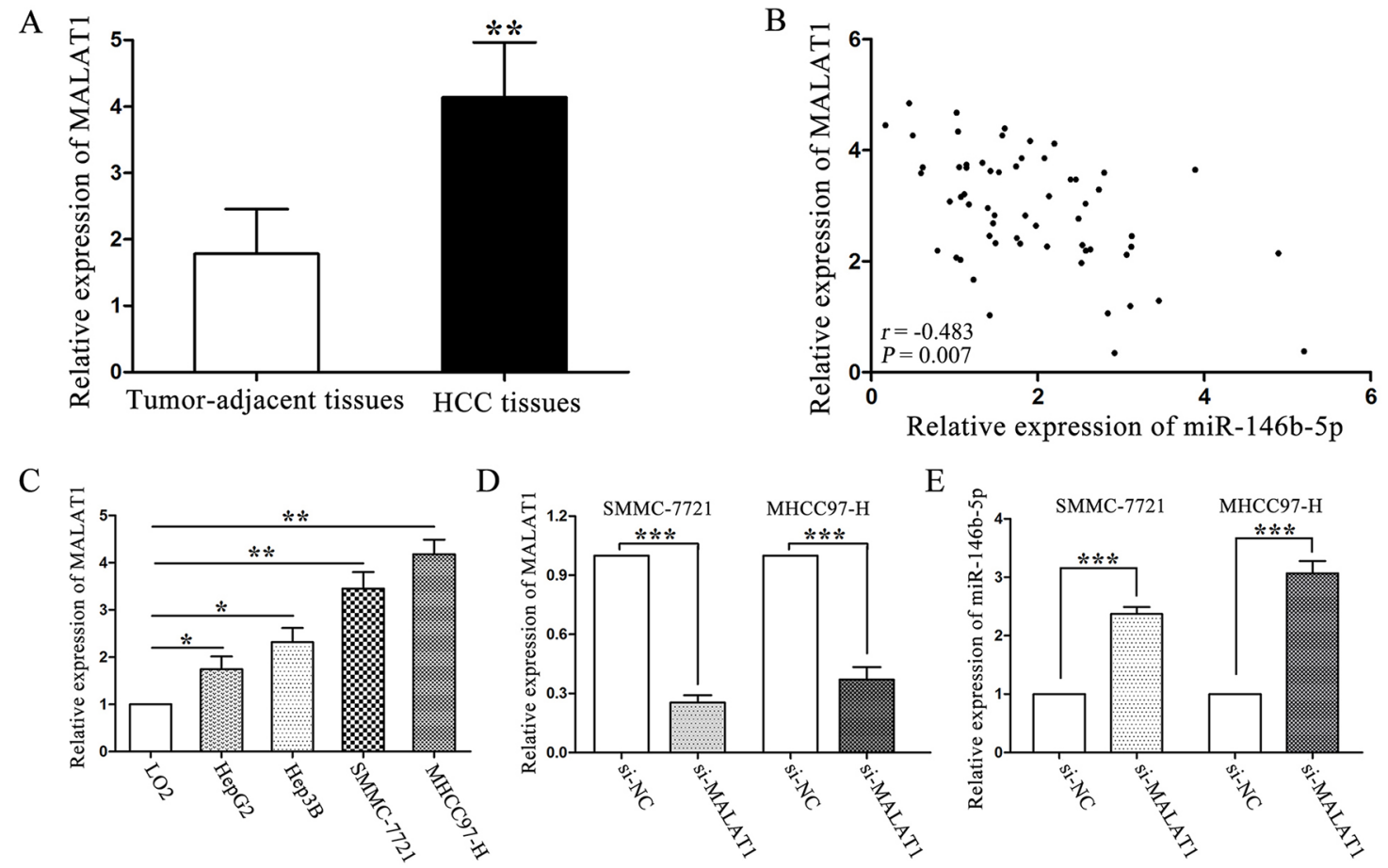

Figure 7: LncRNA MALAT1 negatively regulated miR-146b-5p expression in HCC. A. Expression of MALAT1 in HCC and tumor-adjacent tissues. ${ }^{* *} P<0.01$. B. A negative correlation was verified between MALAT1 and miR-146b-5p in a cohort with $60 \mathrm{HCC}$ patients $(r=-0.483, P=0.007)$. C. The expression of MALAT1 in HCC cells was higher than that in LO2 cells. ${ }^{*} P<0.05,{ }^{* *} P<0.01$. D. Downregulation of MALAT1 by siRNA in SMMC-7721 and MHCC97-H cells. ${ }^{* * *} P<0.001$. E. MALAT1 knockdown increased miR-146b-5p expression in SMMC-7721 and MHCC97-H cells. All experiments were performed at least in triplicate and the data in A, C, D and $\mathbf{E}$ are presented as the (mean $\pm \mathrm{SD})$.

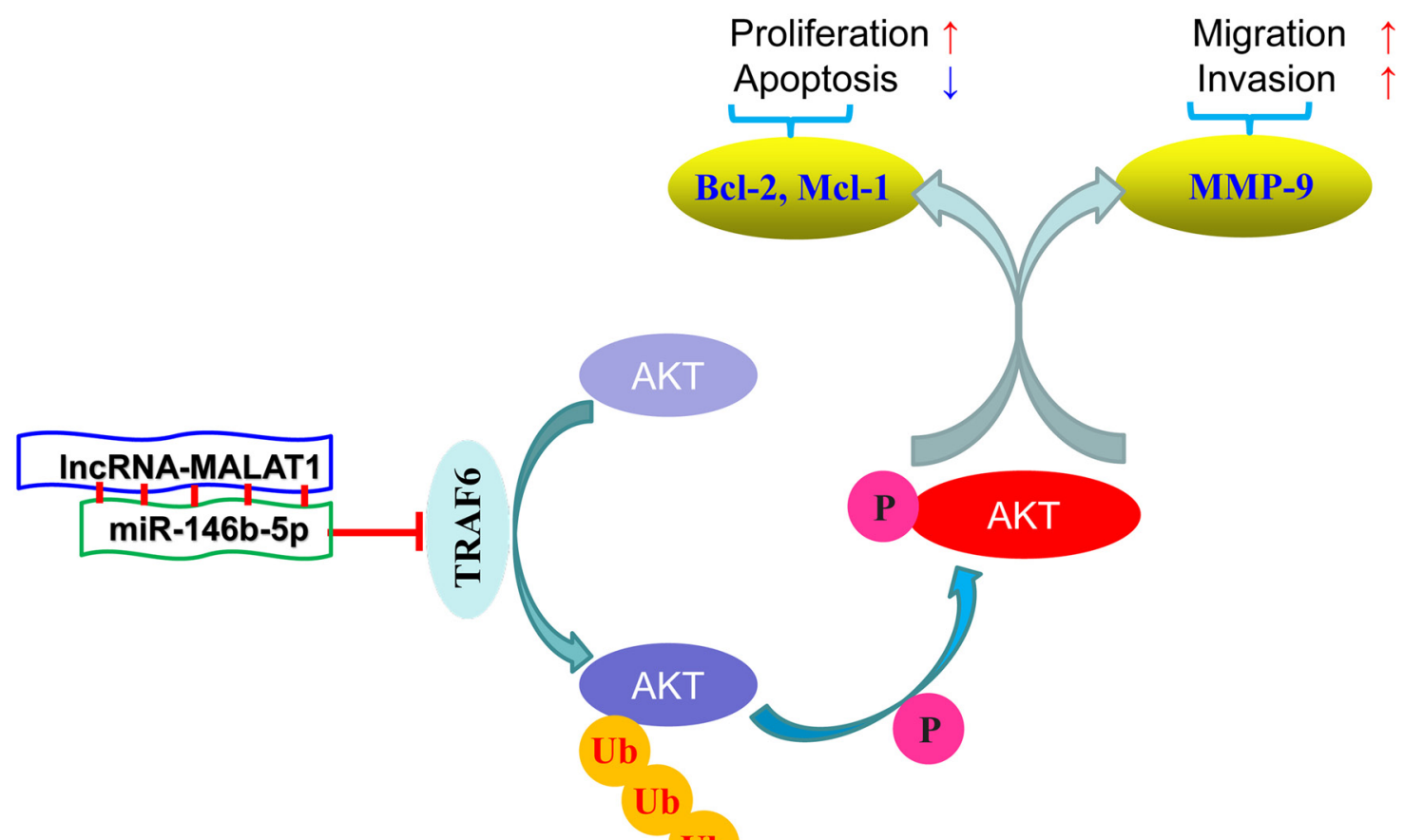

Ub

Figure 8: Diagrammatic sketch of the signaling pathway that miR-146b-5p arrests tumor growth and metastasis on HCC cells. 
To confirm this hypothesis, a series of in vitro and in vivo assays were designed to investigate the biological functions of miR-146b-5p. We revealed that up-regulation of miR-146b-5p reduced proliferation, migration and invasion, promoted apoptosis and caspase 3/7 activity in vitro, and arrested tumor growth and lung metastasis in vivo. Even though miR-146b-5p promoted the proliferation and invasion of osteosarcoma cells [23], our results were still consistent with the previous reports in prostate and pancreatic cancers [24].

Functionally, TRAF6 exhibits an E3 ubiquitin ligase activity. Unlike other E3 ubiquitin ligases catalyze the synthesis of polyubiquitin chains linked through lysine-48 (K48), the chains catalyzed by TRAF6 was linked through the point of lysine-63 (K63) [25]. These unique chains can lead to the activation of TAK1 [26], MAPK [27] and many other kinases' activity. We used dual-luciferase reporter system to confirm that only wildtype miR-146b-5p changed the luciferase activity of 3 'UTR of TRAF6 mRNA. miR-146b-5p could decrease the TRAF6 expression in vitro and in vivo. Finally, we provided a negative correlation between TRAF6 and miR-146b-5p expression in a cohort of HCC patients. Our results also showed that miR-146b-5p only imapired the phosphorylation of Akt through inhibiting the expression of TRAF6 but not influenced the total Akt expression. Because the K48 linked ubiquitination usually caused degradation of target proteins, we could partly confirm that K63 might be the catalytic site of TRAF6 for Akt ubiquitination. We also detected three downstream genes of Akt signaling pathway which could enhance cell growth and metastasis. First, the expression levels of Bcl-2 and Mcl-1 were down-regulated through inhibiting TRAF6/ p-Akt activation by miR-146b-5p. Mcl-1 served as a substrate of caspase-3 [28], its down-regulation could be explained by the enhancement of caspase $3 / 7$ activity after the miR-146b-5p over-expression. Next, our study also found that miR-146b-5p inhibited the expression of MMP9 , and that might explain why miR-146b-5p could inhibit cell invasion and lung metastasis. Furthermore, we rescued the expression of TRAF6 in MHCC97-H $\mathrm{H}^{\text {miR-146b-5p }}$ cells and silenced TRAF6 expression in Hep3B ${ }^{\text {anti-miR-146b-5p }}$ cells. Our results showed that TRAF6 partly abrogated the effects of miR-146b-5p on HCC cell viability, proliferation, apoptosis, migration, invasion through reactivated Akt signaling pathway.

Previous studies showed that deregulation of lncRNAs profoundly influenced the expression of microRNAs [29]. For example, IncRNA HOTAIR contained a conserved binding site for miR-326. Silencing HOTAIR by SiRNA could rescue the expression of miR326 in human glioma cells [30]. Inspired by these studies, we identified a negative correlation between lncRNA MALAT1 and miR-146b-5p expression in HCC tissues. Down-regulation of 1ncRNA MALAT1 expression significantly increased the expression of miR-146b-5p in HCC cells.
In conclusion, this study demonstrated that downregulation of miR-146b-5p in HCC tissues was related to malignant clinical features and poor prognosis. Using in vitro and in vivo studies, miR-146b-5p was demonstrated as a novel inhibitor for tumor growth and metastasis in HCC. The multiple anti-cancer functions of miR-146b-5p were due to the inhibition of the TRAF6/p-Akt pathway (Figure 8). Together, our findings suggested that miR146b-5p could become a novel prognostic bio-marker and potential therapeutic target in HCC.

\section{MATERIALS AND METHODS}

\section{Clinical specimens}

A total of 60 resected HCC tissues and matched tumor-adjacent tissues were provided by the Department of Hepatobiliary Surgery, First Affiliated Hospital of Xi'an Jiaotong University (Xi'an, China) between January 2009 and May 2010. All patients received follow-up after surgery until May 2015. The median follow-up time was 31 months. The demographic, clinical and pathological characteristics were obtained from the medical records and shown in Table 1. Informed consent was obtained in accordance with a protocol approved by the Ethics Committee of Xi'an Jiaotong University.

\section{Cell transfection}

The human immortal liver cell line $\mathrm{LO} 2$ and four HCC cell lines (MHCC97-H, SMMC-7721, Hep3B and HepG2) were obtained from the Institute of Biochemistry and Cell Biology, Chinese Academy of Sciences (Shanghai, China) and cultured with appropriate conditions. miR-146b-5p expression vector (HmiR0164MR04), miRNA control vector (CmiR0001-MR04, miR-NC), miR-146b-5p inhibitor (HmiR-AN0197AM01, anti-miR-146b), miRNA control inhibitor (CmiR-AN0001-AM01, anti-miR-NC) were purchased from GeneCopoeia Co. Ltd. (Guangzhou, China). TRAF6 cDNA clone vector (SC109844) was provided by OriGene Co. Ltd. (Beijing, China). TRAF6 siRNA (s14389), MALAT1 siRNA (4455877) and negative control siRNA (AM4611) were purchased from Applied Biosystems (Foster City, CA, USA). Transfection was performed in a six-well plate when cell confluent attained $70-90 \%$ according to the Lipofectamine ${ }^{\circledR} 2000$ (11668019, Invitrogen, Carlsbad, CA, USA) transfection manual.

\section{Quantitative real-time reverse transcription polymerase chain reaction (qRT-PCR)}

Total RNA was isolated by $\mathrm{TRIZOL}^{\circledR}$ reagent (Invitrogen, Carlsbad, CA, USA). miR-146b-5p BulgeLoop $^{\text {TM }}$ miRNA qRT-PCR Primer Set (miRQ00028091-1) and U6 Bulge-Loop ${ }^{\mathrm{TM}}$ miRNA qRT-PCR Control Primer Set (MQP-0201) were purchased from 
RiboBio Co. Ltd. (Guangzhou, China). The primers of TRAF6 (F: 5'-CTATTCACCAGTTAGAGGG-3'; R: 5'-GCTCACTTACATACATACT-3'), MALAT1 (F: 5'-A TGCGAGTTGTTCTCCGTCT-3'; R: 5'-TATCTGCGG TTTCCTCAAGC-3') and $\beta$-actin (F: 5'-AGAAAATCTGG CACCACACC-3'; R: 5'-AGAGGCGTACAGGGATAG CA-3') were synthesized by Sangon Biotech Co. Ltd. (Shanghai, China). One-Step SYBR ${ }^{\circledR}$ PrimeScript $^{\mathrm{TM}}$ RTPCR Kit (Perfect Real Time, RR066A, TaKaRa, Dalian, China) was used to amplify target genes. $2^{-\Delta \Delta C T}$ method was applied to calculate the relative expression levels. The internal control genes were $\beta$-actin for TRAF6, MALAT1 and U6 snRNA for miR-146b-5p.

\section{In vitro cell viability, proliferation and apoptosis assays}

Cancer cells were seeded into a 96-well plate with $2,000 /$ well in quintuplicate. Cell viability was determined by Cell Counting Kit-8 (CCK-8, E606335, Sangon, Shanghai, China) at $0,24,48$ and 72 hours. Cell proliferation was measured by the plate clone formation assays, and the protocol we described previously [31]. Apoptotic cells were stained at the time of 72 hours' transfection with Annexin V-PE/7-AAD apoptosis detection kit (KGA-1017, KeyGEN, Nangjing, China). $2 \times 10^{5}$ cells were suspended with $50 \mu \mathrm{L}$ binding buffer, and received the following treatments: $5 \mu \mathrm{L} / \mathrm{sample}$ 7-AAD with 15 minutes incubation; $1 \mu \mathrm{L} /$ sample Annexin $\mathrm{V}-\mathrm{PE}$ and $450 \mu \mathrm{L} /$ sample binding buffer with 15 minutes incubation. Positive stained cells were detected by BD FACS Canto II Flow Cytometer (Becton Dickinson, Franklin Lakes, NJ, USA). The caspase-3/7 activity assay was measured using an Apo-ONE ${ }^{\circledR}$ Homogeneous Caspase-3/7 Assay (Promega, Madison, WI, USA) in accordance with our previous description [32].

\section{Transwell chamber models}

The $8 \mu \mathrm{m}$ pore-sized transwell inserts (Nalge Nunc, Penfield, NY, USA) were used to detect cell migration, or coated with matrigel (BD Biosciences, Franklin Lakes, NJ, USA) at $1 \mathrm{mg} / \mathrm{mL}$ on the inner layer to detect cell invasion. Cells were re-suspended with reduced serum DMEM medium (Gibco, Carlsbad, CA, USA) and added into upper-chamber, and a $750 \mu \mathrm{L}$ completed DMEM medium was added into the lower-chamber, then incubating for 24 hours. Cells were fixed in 4\% paraformaldehyde for 2 min and stained with $0.3 \%$ crystal violet. Migrated or invaded cells on the under-surface were counted under a light microscope.

\section{In vivo tumor growth and metastasis assays}

4 weeks BALB/c nude mice were purchased from the center of laboratory animals of Xi'an Jiaotong
University. $5 \times 10^{6}$ cells were suspended in $100 \mu \mathrm{L}$ PBS and injected into the subcutis. Width and length of the neoplasm were measured with calipers every week. Tumor volume was calculated with the formula: $\pi \times$ length $\times$ width $2 / 6.4$ weeks later, mice were excused. 100 mg tissue was collected from every sample to isolate total RNA. Neoplasms were fixed with $4 \%$ paraformaldehyde, embedded in paraffin and made into $4 \mu \mathrm{m}$ slices. Ki-67 antibodies (\#9027, CST, Danvers, MA, USA) were used to determine cell proliferation by immunohistochemical (IHC) staining as we described previously [33]. In situ cell death detection kit, POD (Roche, Mannheim, Germany) was used to detected cell apoptosis according to the manufacturer's instruction. The scores of TUNEL positive staining cells were expressed as the following grades: 0 , $<5 \% ; 1,6 \%-25 \% ; 2,26 \%-50 \% ; 3,51 \%-75 \%$; and 4 , $>75 \%$.

$1 \times 10^{5}$ cells with $100 \mu \mathrm{L}$ PBS were injected into mouse systemic circulation through the tail vein. Lungs were obtained after four weeks feeding and fixed with $4 \%$ paraformaldehyde. $4 \mu \mathrm{m}$ consecutive sections were made and stained with hematoxylin and eosin. The number of metastatic clusters was calculated to analyze the effects of different treatments.

\section{Luciferase reporter assay}

The 3'-UTR region of TRAF6 was inserted into pmiR-RB-Report $^{\mathrm{TM}}$ vector (RiboBio, Guangzhou, China). miR-146b-5p and recombinant vectors were co-transfected into MHCC97-H cells with Lipofectamine ${ }^{\circledR} 2000$. Ranilla luciferase activity was detected using the dual-luciferase reporter assay system (Promega, Madison, WI, USA) to reflect the binding between miR-146b-5p and the 3'-UTR region of TRAF6. Firefly luciferase activity was served as the internal control.

\section{Immunoblotting}

Cells were dissolved by RIPA reagent (HEART Biotech, Xi'an, China). Protein levels were quantified by BCA protein assay kit II (\#5000002, BIO-RAD, Hercules, CA, USA). Lysates (40 $\mu \mathrm{g}$ protein) were separated by $10 \%$ SDS-PAGE gel and transferred onto a PVDF membrane (IPVH00010, Millipore, Billerica, MA, USA). Primary antibodies of TRAF6, Akt (\#4691), Phospho$\mathrm{Akt}^{\mathrm{Thr} 308}$ (\#13038) and $\beta$-actin (\#3700) were purchased from CST (Danvers, MA, USA). Primary antibodies of Bcl-2 (12789-1-AP), Mcl-1 (16225-1-AP) and MMP9 (10375-2-AP) were purchased from PROTEINTECH (Rosemont, IL, USA). PVDF membranes with certain proteins were incubated with matched antibodies (diluted as 1:1000) overnight. Secondary horseradish peroxidaseconjugated anti-rabbit or anti-mouse antibodies (diluted as 1:5000, ABGENT, San Diego, CA, USA) were used to bind matched primary antibodies. Protein expression 
levels were visualized by Enhanced chemiluminescence reagent (WBKLS0500, Millipore, Billerica, MA, USA) and calculated by Image $\mathbf{J}$ software.

\section{Statistical analysis}

Measurement data is presented as Mean $\pm \mathrm{SD}$ and processed by the SPSS statistical package for Window Version 21 (SPSS Inc., Chicago, IL, USA) and GraphPad Prism 5 software (GraphPad Inc., San Diego, CA, USA). Student's t test or one-way ANOVA were used to analyze the difference among/between sample groups. Patients' survival was reflected by Kaplan-Meier and analyzed by log-rank test. Person correlation analysis was used to evaluate the relationship between miR-146b-5p and MALAT1. $P<0.05$ was considered to be statistically significant.

\section{Author contributions}

Chao Li: Designed the research and wrote the paper; Runchen Miao, Sushun Liu, Simin Zhang, Yan Deng, Jianbin Bi: Participated in research work; Yong Wan: Constructed figures; Kai Qu, Jingyao Zhang: Revised the paper; Chang Liu: Designed the research.

\section{CONFLICTS OF INTEREST}

All authors declare no conflicts of interest.

\section{FUNDINGS}

This work was supported by a grant from the National Natural Scientific Foundation of China (NO.81472247).

\section{REFERENCES}

1. Zeng H, Zheng R, Guo Y, Zhang S, Zou X, Wang N, Zhang L, Tang J, Chen J, Wei K, Huang S, Wang J, Yu L, et al. Cancer survival in China, 2003-2005: a population-based study. International journal of cancer. 2015; 136:1921-30.

2. Su Z, Yang Z, Xu Y, Chen Y, Yu Q. MicroRNAs in apoptosis, autophagy and necroptosis. Oncotarget. 2015; 6:8474-90. doi: 10.18632/oncotarget.3523.

3. Jia YY, Zhao JY, Li BL, Gao K, Song Y, Liu MY, Yang XJ, Xue Y, Wen AD, Shi L. miR-592/WSB1/HIF-1 $\alpha$ axis inhibits glycolytic metabolism to decrease hepatocellular carcinoma growth. Oncotarget. 2016; 7:35257-69. doi: 10.18632/oncotarget.9135.

4. Tu K, Li C, Zheng X, Yang W, Yao Y, Liu Q. Prognostic significance of miR-218 in human hepatocellular carcinoma and its role in cell growth. Oncology reports. 2014; 32:1571-7.

5. Xu X, Chen W, Miao R, Zhou Y, Wang Z, Zhang L, Wan Y, Dong Y, Qu K, Liu C. miR-34a induces cellular senescence via modulation of telomerase activity in human hepatocellular carcinoma by targeting FoxM1/c-Myc pathway. Oncotarget. 2015; 6:3988-4004. doi: 10.18632/ oncotarget.2905.

6. Yang X, Zhang XF, Lu X, Jia HL, Liang L, Dong QZ, Ye QH, Qin LX. MicroRNA-26a suppresses angiogenesis in human hepatocellular carcinoma by targeting hepatocyte growth factor-cMet pathway. Hepatology. 2014; 59:1874-85.

7. Zhou SL, Hu ZQ, Zhou ZJ, Dai Z, Wang Z, Cao Y, Fan J, Huang XW, Zhou J. miR-28-5p-IL-34-macrophage feedback loop modulates hepatocellular carcinoma metastasis. Hepatology. 2016; 63:1560-75.

8. Squadrito ML, Etzrodt M, De Palma M, Pittet MJ. MicroRNA-mediated control of macrophages and its implications for cancer. Trends in immunology. 2013; $34: 350-9$

9. Xu E, Zhao J, Ma J, Wang C, Zhang C, Jiang H, Cheng J, Gao R, Zhou X. miR-146b-5p promotes invasion and metastasis contributing to chemoresistance in osteosarcoma by targeting zinc and ring finger 3. Oncology reports. 2016; 35:275-83

10. Li Y, Wang Y, Yu L, Sun C, Cheng D, Yu S, Wang Q, Yan Y, Kang C, Jin S, An T, Shi C, Xu J, et al. miR-146b-5p inhibits glioma migration and invasion by targeting MMP16. Cancer letters. 2013; 339:260-9.

11. Yang W, Yu H, Shen Y, Liu Y, Yang Z, Sun T. MiR-146b-5p overexpression attenuates stemness and radioresistance of glioma stem cells by targeting HuR/lincRNA-p21/ $\beta$-catenin pathway. Oncotarget. 2016; 7:41505-26. doi: 10.18632/ oncotarget.9214.

12. Peng Y, Li C, Luo DC, Ding JW, Zhang W, Pan G. Expression profile and clinical significance of microRNAs in papillary thyroid carcinoma. Molecules. 2014; 19:11586-99.

13. Guo Z, Hardin H, Montemayor-Garcia C, Asioli S, Righi A, Maletta F, Sapino A, Lloyd RV. In Situ Hybridization Analysis of miR-146b-5p and miR-21 in Thyroid Nodules: Diagnostic Implications. Endocrine pathology. 2015; 26:157-63.

14. Lima CR, Geraldo MV, Fuziwara CS, Kimura ET, Santos MF. MiRNA-146b-5p upregulates migration and invasion of different Papillary Thyroid Carcinoma cells. BMC cancer. 2016; 16:108. doi: 10.1186/s12885-016-2146-z.

15. Geraldo MV, Yamashita AS, Kimura ET. MicroRNA miR146b-5p regulates signal transduction of TGF-beta by repressing SMAD4 in thyroid cancer. Oncogene. 2012; 31:1910-22.

16. Lee JC, Zhao JT, Gundara J, Serpell J, Bach LA, Sidhu S. Papillary thyroid cancer-derived exosomes contain miRNA146b and miRNA-222. The Journal of surgical research. $2015 ; 196: 39-48$.

17. Liu W, Qi Y, Liu L, Tang Y, Wei J, Zhou L. Suppression of tumor cell proliferation by quinine via the inhibition of 
the tumor necrosis factor receptorassociated factor 6AKT interaction. Molecular medicine reports. 2016; 14:2171-9.

18. Cai J, Xu L, Cai Z, Wang J, Zhou B, Hu H. MicroRNA$146 b-5 p$ inhibits the growth of gallbladder carcinoma by targeting epidermal growth factor receptor. Molecular medicine reports. 2015; 12:1549-55.

19. Zhao Y, Schetter AJ, Yang GB, Nguyen G, Mathe EA, Li P, Cai H, Yu L, Liu F, Hang D, Yang H, Wang XW, Ke $\mathrm{Y}$, et al. microRNA and inflammatory gene expression as prognostic marker for overall survival in esophageal squamous cell carcinoma. International journal of cancer. 2013; 132:2901-9.

20. Wu PY, Zhang XD, Zhu J, Guo XY, Wang JF. Low expression of microRNA-146b-5p and microRNA-320d predicts poor outcome of large B-cell lymphoma treated with cyclophosphamide, doxorubicin, vincristine, and prednisone. Human pathology. 2014; 45:1664-73.

21. Zhou L, Zhao X, Han Y, Lu Y, Shang Y, Liu C, Li T, Jin Z, Fan D, Wu K. Regulation of UHRF1 by miR-146a/b modulates gastric cancer invasion and metastasis. FASEB journal. 2013; 27:4929-39. doi: 10.1096/fj.13-233387.

22. Yoon SO, Kim EK, Lee M, Jung WY, Lee H, Kang Y, Jang YJ, Hong SW, Choi SH, Yang WI. NOVA1 inhibition by miR-146b-5p in the remnant tissue microenvironment defines occult residual disease after gastric cancer removal. Oncotarget. 2016; 7:2475-95. doi: 10.18632/ oncotarget.6542.

23. Al-Khalaf HH, Aboussekhra A. MicroRNA-141 and microRNA-146b-5p inhibit the prometastatic mesenchymal characteristics through the RNA-binding protein AUF1 targeting the transcription factor ZEB1 and the protein kinase AKT. The Journal of biological chemistry. 2014; 289:31433-47.

24. Lin F, Wang X, Jie Z, Hong X, Li X, Wang M, Yu Y. Inhibitory effects of miR-146b-5p on cell migration and invasion of pancreatic cancer by targeting MMP16. Journal of Huazhong University of Science and Technology Medical sciences. 2011; 31:509-14.

25. Min Y, Wi SM, Kang JA, Yang T, Park CS, Park SG, Chung S, Shim JH, Chun E, Lee KY. Cereblon negatively regulates
TLR4 signaling through the attenuation of ubiquitination of TRAF6. Cell death \& disease. 2016; 7:e2313.

26. Ji YX, Zhang P, Zhang XJ, Zhao YC, Deng KQ, Jiang X, Wang PX, Huang Z, Li H. The ubiquitin E3 ligase TRAF6 exacerbates pathological cardiac hypertrophy via TAK1dependent signalling. Nature communications. 2016; 7:11267.

27. Stack J, Hurst TP, Flannery SM, Brennan K, Rupp S, Oda S, Khan AR, Bowie AG. Poxviral protein A52 stimulates p38 mitogen-activated protein kinase (MAPK) activation by causing tumor necrosis factor receptor-associated factor 6 (TRAF6) self-association leading to transforming growth factor beta-activated kinase 1 (TAK1) recruitment. The Journal of biological chemistry. 2013; 288:33642-53.

28. Hu L, Chen L, Yang G, Li L, Sun H, Chang Y, Tu Q, Wu M, Wang H. HBx sensitizes cells to oxidative stress-induced apoptosis by accelerating the loss of Mcl-1 protein via caspase-3 cascade. Molecular cancer. 2011; 10:43.

29. Liz J, Esteller M. lncRNAs and microRNAs with a role in cancer development. Biochimica et biophysica acta. 2016; 1859:169-76.

30. Ke J, Yao YL, Zheng J, Wang P, Liu YH, Ma J, Li Z, Liu XB, Li ZQ, Wang ZH, Xue YX. Knockdown of long non-coding RNA HOTAIR inhibits malignant biological behaviors of human glioma cells via modulation of miR326. Oncotarget. 2015; 6:21934-49. doi: 10.18632/ oncotarget.4290.

31. Tu K, Zheng X, Zhou Z, Li C, Zhang J, Gao J, Yao Y, Liu Q. Recombinant human adenovirus-p53 injection induced apoptosis in hepatocellular carcinoma cell lines mediated by $553-F b x w 7$ pathway, which controls c-Myc and cyclin E. PloS one. 2013; 8:e68574.

32. Tu K, Yang W, Li C, Zheng X, Lu Z, Guo C, Yao Y, Liu Q. Fbxw7 is an independent prognostic marker and induces apoptosis and growth arrest by regulating YAP abundance in hepatocellular carcinoma. Molecular cancer. 2014; 13:110.

33. Li C, Yang W, Zhang J, Zheng X, Yao Y, Tu K, Liu Q. SREBP-1 has a prognostic role and contributes to invasion and metastasis in human hepatocellular carcinoma. International journal of molecular sciences. 2014; 15:7124-38. 